\title{
Semiclassical Quantization Rule for Bound-State Spectrum in Quantum Dots: Scattering Phase Approximation
}

\author{
Wei Chen \\ Department of Physics, National Tsing-Hua University, Hsinchu 300, Taiwan, R.O.C. \\ Tzay-Ming Hong \\ Department of Physics, University of Texas, Austin, TX 78712, U.S.A. and \\ Department of Physics, National Tsing-Hua University, Hsinchu 300, Taiwan, R.O.C. \\ Hsiu-Hau Lin \\ Department of Physics, National Tsing-Hua University, Hsinchu 300, Taiwan, R.O.C. and \\ Physics Division, National Center for Theoretical Sciences, Hsinchu 300, Taiwan, R.O.C.
}

(Dated: October 31, 2018)

\begin{abstract}
We study the quantum propagator in the semiclassical limit with sharp confining potentials. Including the energy-dependent scattering phase due to sharp confining potential, the modified Van Vleck's formula is derived. We also discuss the close relations among quantum statistics, discrete gauge symmetry, and hard-wall constraints. Most of all, we formulate a new quantization rule that applies to both smooth and sharp boundary potentials. It provides an easy way to compute quantized energies in the semiclassical limit and is extremely useful for many physical systems.
\end{abstract}

\section{INTRODUCTION}

The most straightforward method to obtain the boundstate spectrum for a quantum system is to solve the Schrödinger equation. However, if the potential profile is smooth compared with the wavelength of the particle, the energy spectrum can be obtained by the semiclassical WKB approximation. The semiclassical approach reduces the task of solving the differential equation into a simple integral. While the great simplification is attractive, it does not work all the times. In quantum dots, the confining potential is usually sharp and leads to strong quantum interferences which invalidate the semiclassical approach. One notices that the semiclassical approximation only breaks down near the sharp confining potential. It motivates us to generalize the conventional semiclassical approach by including the quantum interferences exactly near the turning points where the semiclassical approximation is not appropriate. Rather nicely, we were able to capture the complicated quantum interference effects by a simple energy-dependent scattering phase correction.

To elucidate this point, it is convenient to adapt the path integral formulism. Path integral provides an alternative approach to formulate quantum mechanics [1, 2]. The quantum propagator $G\left(x, x^{\prime} ; T\right)$, that is the key quantity in quantum mechanics, is shown to equal the summation over all possible paths with the same end points. In the semiclassical limit, the dominant contribution comes from classical trajectories and fluctuations around them 3 , 4, 5]. Within the stationary phase approximation including fluctuations up to the quadratic order, the quantum propagator can be approximated by the Van Vleck's formula [6]. In general, there would be many classical trajectories that satisfies the same boundary conditions, and the Berry phase interferences be- tween them are important 7, 8, 9]. By Morse's theorem, the second variation, considered as quadratic fluctuations around a given trajectory from $x^{\prime}$ to $x$ in time $T$, has as many negative eigenvalues as there are conjugate (turning) points along the trajectory. These conjugate points give rise to a Berry phase $\nu \pi / 2$ for the trajectory, where $\nu$ is the total number of conjugate points along the trajectory, or sometimes referred to as the Maslov or Morse index 10 .

Not only elucidating the crossover between classical and quantum mechanics, the semiclassical limit also provides a convenient way to calculate the bound state energy. Instead of solving the Schrödinger equation directly, the bound state spectrum can also be computed by the WKB approximation 11]. In order to account for the interference effects among classical trajectories correctly, we rederive Van Vleck's formula with an extra scattering phase correction due to sharp confining potentials.

Following the standard stationary phase approximation and making a Legendre transformation of the time variable in the quantum propagator to the energy variable, we are able to generalize the Einstein-BrillouinKeller (EBK) quantization rule 12, 13, 14] with an additional phase correction term

$$
\oint \sqrt{2 m[E-V(x)]} d x=2 n \pi+\sum_{s} \phi_{s}(E)
$$

where $\phi_{s}(E)$ is the energy-dependent scattering phase due to collisions with the confining potential. The usual WKB approximation is the special case where the scattering phase at each turning point is assume to take on the energy-independent value $\phi_{s}(E)=\pi / 2$. On the other hand, if the confining potential becomes infinitely sharp (hard-wall limit), the scattering phase rises to $\pi$. The modified EBK quantization rule in Eq. (11) relaxes the requirement of the potential smoothness in the WKB 
approximation. This is of great advantage because many physical systems including quantum dots, quantum wells, Hall bars, electronic wave guides, etc., have both hardwall-like potentials (from sample edges) as well as smooth potentials (by applying external fields) at the same time.

The paper is organized in the following way. In Sec. II, we introduce the Van Vleck's formula and apply it to simple systems. We explicitly show that the Van Vleck's approximation is incorrect in the presence of hard walls and the scattering phase correction is crucial. In Sec III, we compute the energy dependence of the scattering phase at each turning point and derive the modified Van Vleck's formula. In Sec. IV, we derive the key result of this paper - the modified EBK quantization rule. We apply it to physical systems with both smooth and hard confinement potentials and show that the modified term is necessary to obtain the correct energy levels. Finally, in Sec V, we relate the connection between the quantum statistics, discrete gauge symmetry to the scattering phase approach. Then a brief conclusion follows.

\section{QUANTUM PROPAGATOR AND CLASSICAL TRAJECTORIES}

In the path integral formalism [1], the quantum propagator equals the sum over all possible paths with the same end points,

$$
\begin{aligned}
G\left(x, x^{\prime} ; T\right) & \equiv\left\langle x\left|e^{-i H T}\right| x^{\prime}\right\rangle \\
& =\int_{x^{\prime}}^{x} \mathcal{D}[x] \exp \left(i \int_{0}^{T} L(x, \dot{x}, t) d t\right),
\end{aligned}
$$

where the measure $\mathcal{D}[x]$ denotes all possible paths with end points $x(0)=x^{\prime}$ and $x(T)=x$. In the semiclassical limit, the phase inside the path integral oscillates rapidly except in the neighborhood of the classical trajectories. Within the stationary phase approximation including fluctuations up to quadratic order, the propagator is approximated by the Van Vleck's formula,

$$
G\left(x, x^{\prime} ; T\right) \simeq \frac{1}{\sqrt{2 \pi i}} \sum_{p} \sqrt{C_{p}} \exp \left[i A_{p}-i \nu_{p} \frac{\pi}{2}\right],
$$

where $A_{p}\left(x, x^{\prime} ; T\right)$ is the action of the classical trajectory starting from $x(0)=x^{\prime}$ and ending at $x(T)=x$, and the subscript $p$ denotes all classical paths with the desired end points. The strength of the quadratic fluctuations 8$]$ around the classical trajectory is

$$
C_{p}=\left|-\frac{\partial^{2} A}{\partial x \partial x^{\prime}}\right| \text {. }
$$

Finally, the total number of conjugate (or turning) points along the classical trajectory is denoted by $\nu$. Notice that, for each conjugate point, there is a $\pi / 2$ Berry phase associated with it. Van Vleck's formula provides a completely classical approximation of the quantum propagator, in the sense that all relevant elements can be computed from the classical trajectories.
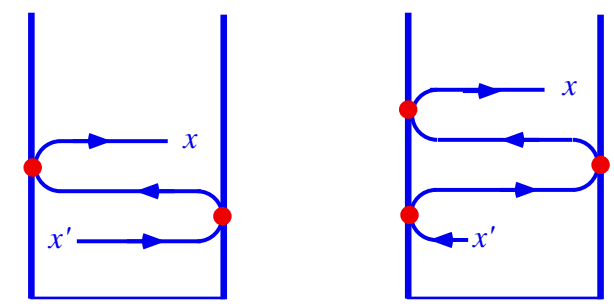

FIG. 1: Classical trajectories in the presence of two hard walls. On the left is a trajectory with even reflection points $r=2$, while the right with odd reflection points $r=3$.

A straightforward example of the Van Vleck's formula is a free particle moving on the a finite ring with length $L$. There are infinite classical paths which satisfy the conditions $x(0)=x^{\prime}$ and $x(T)=x$. The total (route) distance of each classical trajectory is $d_{n}=x-x^{\prime}+n L$, where $n$ is an integer. The action for each trajectory is

$$
A_{n}\left(x, x^{\prime} ; T\right)=\frac{m}{2 T}\left(x-x^{\prime}+n L\right)^{2} .
$$

Taking the derivative of the action, the strength of fluctuations around each trajectory $C_{n}=m / T$ is independent of the end points and the choice of trajectories. Since the particle moves at constant velocity, it is obvious that there is no conjugate point along any classical trajectory and thus $\nu_{n}=0$. Besides, because the fluctuations of the classical trajectory of a free particle are exactly quadratic, we expect the Van Vleck's formula to be exact for this system,

$$
G(x, x ; T)=\sqrt{\frac{m}{2 \pi i T}} \sum_{n} \exp \left[i \frac{m}{2 T}\left(x-x^{\prime}+n L\right)^{2}\right]
$$

This infinite sum can be re-written in terms of its Fourier function with the use of Poisson summation formula in Appendix A. 15. Notice that

$$
f(y)=e^{i \alpha(y+\beta)^{2}} \leftrightarrow F(p)=\sqrt{\frac{i \pi}{\alpha}} e^{-i k^{2} / 4 \alpha+i k \beta}
$$

Choosing $a=L$, the summation over coordinate $y=n a$ can be turned into the summation over momentum $k_{n}=$ $2 n \pi / L$. The propagator is then

$$
G(x, x ; T)=\frac{1}{L} \sum_{n} \exp \left[i k_{n}\left(x-x^{\prime}\right)-i E_{n} T\right],
$$

where $k_{n}=2 n \pi / L$ is the quantized momentum and $E_{n}=k_{n}^{2} / 2 m$ is the quantized energy. It is obvious that the propagator $G\left(x, x^{\prime} ; T\right)$ calculated by the Van Vleck's formula is exact in this case.

Let us now apply the Van Vleck's formula to another physical system - a free particle bouncing back and forth between two hard walls. We calculate the propagator explicitly and show that the Van Vleck's formula leads to incorrect results. 
The trajectories in this problem can be classified by the number of collisions with the hard walls, as seen in Fig. 1. For those trajectories that collide with the hard walls even times, the route distance is $d_{n}^{e}=x-x^{\prime}+2 n L$, while the distance is $d_{n}^{o}=x+x^{\prime}+2 n L$ for trajectories that collide with the walls odd times. The action for each trajectory can be computed straightforwardly

$$
\begin{aligned}
& A_{n}^{e}\left(x, x^{\prime} ; T\right)=\frac{m}{2 T}\left(x-x^{\prime}+2 n L\right)^{2}, \\
& A_{n}^{o}\left(x, x^{\prime} ; T\right)=\frac{m}{2 T}\left(x+x^{\prime}+2 n L\right)^{2} .
\end{aligned}
$$

Here $A^{e / o}\left(x, x^{\prime} ; T\right)$ denotes the action for trajectories with even/odd reflection points. The fluctuations along all trajectories contribute the same $C_{n}=m / T$ as in the previous example. For an one-dimensional motion, a conjugate point is identified as the position where the velocity vanishes. However, for a free particle bouncing back and forth between two hard walls, the velocity is constant up to a minus sign and does not vanish at any point along the classical trajectory. Thus, the number of conjugate points is zero, $\nu=0$.

The propagator without any phase correction is

$$
\begin{aligned}
G_{V V}\left(x, x^{\prime} ; T\right) & =\sqrt{\frac{m}{2 \pi i T}} \sum_{n}\left\{\exp \left[i \frac{m}{2 T}\left(x-x^{\prime}+2 n L\right)^{2}\right]\right. \\
& \left.+\exp \left[i \frac{m}{2 T}\left(x+x^{\prime}+2 n L\right)^{2}\right]\right\}
\end{aligned}
$$

Both infinite sums can be turned into summations over discrete momentum again by mean of the Poisson summation formula. The prefactors cancel as in the previous example and we are left with the simple result,

$$
\begin{aligned}
& G_{V V}\left(x, x^{\prime} ; T\right)=\frac{1}{L} \sum_{n=0}^{\infty} \exp \left[-i E_{n} t\right] \times \\
& \quad \times\left\{\cos \left[k_{n}\left(x-x^{\prime}\right)\right]+\cos \left[k_{n}\left(x+x^{\prime}\right)\right]\right\},
\end{aligned}
$$

where $k_{n}=n \pi / L$ is the quantized momentum and $E_{n}=$ $k_{n}^{2} / 2 m$ is the quantized energy. Combining two cosines would leads to $\cos \left(k_{n} x\right) \cos \left(k_{n} x^{\prime}\right)$, while the correct form should be $\sin \left(k_{n} x\right) \sin \left(k_{n} x^{\prime}\right)$. In fact, one can recover the exact answer (with all prefactors right!) if we change the sign of the second term in Eq. (12). That is, only if we assign an extra phase $\pi$ to trajectories with odd reflection points, will the modified Van Vleck's formula become correct!

In the following section, we study the path integral formalism in the presence of a single hard-wall boundary and show that an extra phase correction arises naturally due to collisions with the confining potential.

\section{SCATTERING PHASE DUE TO HARD WALL}

Consider a particle moving under the influence of a regular potential $V(x)$ and a hard-wall potential $V_{c}(x)$.
The Hamiltonian is

$$
H=\frac{p^{2}}{2 m}+V(x)+V_{c}(x),
$$

where $V_{c}(x)$ is the hard-wall potential at $x=0$,

$$
V_{c}(x)=\left\{\begin{array}{cc}
0, & x>0 \\
\infty, & x<0
\end{array}\right.
$$

The regular potential is treated in the ordinary way while the hard-wall one is viewed as the depletion of Hilbert space. The complete set of the Hilbert space is now reduced,

$$
\begin{aligned}
\int_{0}^{\infty} d r|r\rangle\langle r| & =\mathbf{1} \\
\sum_{\phi=0, \pi} \int \frac{d p}{2 \pi} e^{i \phi}|p\rangle\left\langle e^{i \phi} p\right| & =\mathbf{1}
\end{aligned}
$$

It would become clear later that the phase $\phi$ is associated with the scattering phase in the path integral. Slicing the time interval $T$ into infinitesimal pieces and inserting complete sets of the coordinate space, the propagator is

$$
\begin{aligned}
G\left(r, r^{\prime} ; T\right) & =\left\langle r\left|e^{-i H T}\right| r^{\prime}\right\rangle \\
& =\int_{0}^{\infty} d r_{n} \prod_{n=0}^{N-1}\left\langle r_{n+1}\left|e^{-i \epsilon H}\right| r_{n}\right\rangle
\end{aligned}
$$

where $r_{N}=r$ and $r_{0}=r^{\prime}$ are all positive. Each matrix element in the product is computed by inserting the complete set in momentum space into Eq. [16),

$$
\begin{aligned}
\left\langle r_{n+1}\right| e^{-i \epsilon H} & \left|r_{n}\right\rangle=\int \frac{d p_{n}}{2 \pi} \exp \left[-i \epsilon H_{n}\right] \\
\times & \sum_{x_{n}= \pm r_{n}} e^{i p_{n}\left(r_{n+1}-x_{n}\right)-i \phi_{n}}
\end{aligned}
$$

where the phase $\phi=0$ for $x_{n}=r_{n}$, and $\phi=\pi$ when $x_{n}=-r_{n}$. Since $x_{n}= \pm r_{n}$, the two terms can be combined and lead to the unconstraint integral over $x_{n}$. After changing the constrained variable $r_{n}$ to $x_{n}$, it is convenient to write the Berry phase $\phi_{n}$ in the following way

$$
\phi_{n}=\pi\left[\Theta\left(x_{n+1}\right)-\Theta\left(x_{n}\right)\right] .
$$

Notice that the Berry phase is zero if the path does not pass through $x=0$ in the infinitesimal time interval $d t_{n}$ and $\pi$ if the path passes through. The integral over momentum can be carried out easily and the propagator is

$$
G\left(r, r^{\prime} ; T\right)=\sum_{x^{\prime}= \pm r^{\prime}} e^{i \phi_{s}} \int_{x^{\prime}}^{r} \mathcal{D}[x] \exp \left[i \mathcal{A}\left(r, x^{\prime} ; T\right)\right]
$$

The total phase $\phi_{s}=\pi\left[\Theta(r)-\Theta\left(x^{\prime}\right)\right]$ is a boundary term and can be pulled out of the path integral [2]. The paths are divided into two topologically distinct classes. For all possible paths starting from $r$ to $r^{\prime}$, the scattering 
phase is zero, while for those starting from $r$ to $-r^{\prime}$, the scattering phase is $\pi$ that causes a minus sign.

The classical trajectories among the paths can be then classified in the same way. Furthermore, trajectories with end points $r$ and $r^{\prime}$ can be identified as trajectories (in the physical half plane) with even reflection points and those with end points $r$ and $-r^{\prime}$ are trajectories with odd reflection points. Therefore, in the semiclassical limit, the Van Vleck's formula is modified with an extra phase term,

$$
G\left(r, r^{\prime} ; T\right) \simeq \frac{1}{\sqrt{2 \pi i}} \sum_{p} \sqrt{C_{p}} \exp \left[i A_{p}-i \phi_{p}\right],
$$

The proof for more than one turning point is straightforward and the scattering phase just add up. It would become clear in the following section that the scattering phase correction is crucially important in determine the energy spectrum.

\section{MODIFIED EBK QUANTIZATION RULE}

The most powerful use of Van Vleck's formula is that it leads to the EBK quantization rule in the semiclassical limit. One notices that, if we set $x=x^{\prime}$ in the propagator and integrate over all possible $x$, it results in the quantum partition function $Z(T)=\sum_{n} \exp \left[-i E_{n} T\right]$. The energy levels can then be identified as the singularities of $Z(\omega)$ which is the Fourier transformation of the partition function. Within stationary phase approximation, it can be shown that the total phase $\oint p d q-i \nu \pi / 2$ (in the absence of sharp boundaries) is quantized [2] and leads to the EBK quantization rule,

$$
\oint p d q=2 n \pi+\nu \frac{\pi}{2}
$$

where $\nu$ is the number of turning points along the periodic orbit. The usual WKB approximation is the special case with two conjugate points $\nu=2$. The presence of the sharp boundaries changes the scattering phase at each turning point from $\pi / 2$ to $\pi$ and leads to the modified EBK quantization rule. It is interesting to see that the scattering due to sharp confining potential modified the spectrum only through the scattering phase $\phi_{s}$.

Now we are ready to consider the confining potential in more general form

$$
V_{c}(x)=\Theta(-x)\left[V_{0}+V_{1}|x|\right],
$$

where $V_{0} \equiv k_{0}^{2} / 2 m$ is the potential height and $V_{1}=$ $k_{1}^{3} / 2 m$ is the slope of the confining potential. The scattering due to $V_{c}(x)$ can be solved exactly and the eigenstates are

$$
|\psi(k)\rangle=|k\rangle+e^{-i \phi_{s}(k)}|-k\rangle .
$$

The scattering phase is apparently energy-dependent as shown in Figure 2. For the hard-wall potential $\left(k / k_{0}=\right.$

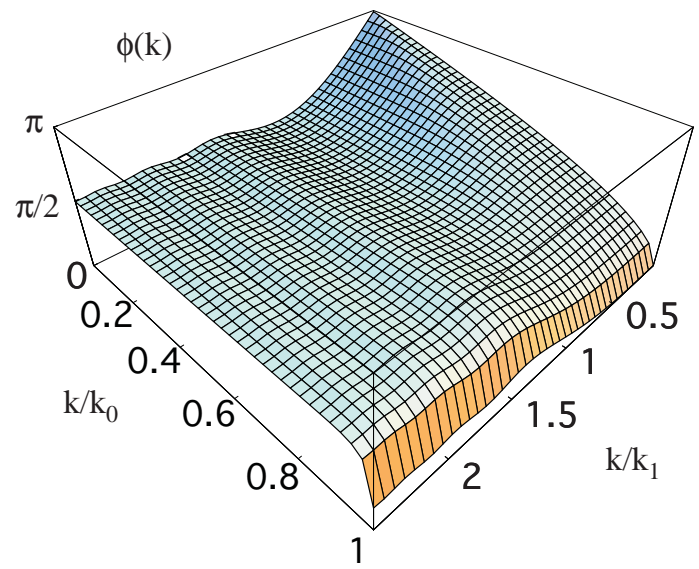

FIG. 2: Scattering phase for different potential height $V_{0}=$ $k_{0}^{2} / 2 m$ and slop $V_{1}=k_{1}^{3} / 2 m$.

$\left.0, k / k_{1}=0\right)$, the scattering phase is $\pi$, while for the smooth potential $\left(k / k_{0} \ll 1, k / k_{1} \gg 1\right)$ the phase becomes $\pi / 2$ as in the WKB approximation. Following similar calculation in previous section, we arrive at the modified EBK quantization integral in Eq. 1]

We apply the modified EBK quantization rule to a finite potential well of length $L$ and with height $V_{0}=$ $k_{0}^{2} / 2 m$. After some algebra, the scattering phase is shown to be $\phi(k)=2 \cos ^{-1}\left[\left(k / k_{0}\right)^{2}-1\right]$. The quantized energy $E_{n}=k_{n}^{2} / 2 m$ satisfies

$$
2 k_{n} L=2 n \pi+\phi\left(k_{n}\right) .
$$

Quite surprisingly, the spectrum obtained by the semiclassical approach is identical to the exact solution. This shows that the quantum interference effects arose from the sharp confining potential can be captured by the scattering phase rather well.

The modified EBK quantization rule can also be applied to physical systems in higher dimensions. Let us consider a spherical or hemispherical qunatum dot. We can either apply the modified EBK formula directly to the true three-dimensional trajectories 18 or apply the formula after reducing the system to one dimension. Here we adapt the second approach. After separation of variables, the radial effective Hamiltonian of the threedimensional spherical (hemispherical) quantum dot becomes one-dimensional with the effective potential

$$
V=\left\{\begin{array}{cc}
\frac{l(l+1)}{2 m r^{2}}, & r<a \\
\infty, & r>a
\end{array},\right.
$$

where $l$ is the quantized angular momentum. For the spherical quantum dot, $l$ takes on all integer values, while for the hemispherical dot, only odd integers are allowed due to the flat boundary.

The classical trajectory of the electron is confined between the hard-wall boundary at the surface and the centrifugal potential near the origin. Thus, there are one reflection point $\phi_{s}=\pi$ and one conjugate point $\phi_{s}=\pi / 2$. 
Applying the modified EBK quantization rule, the approximate energy satisfies the algebraic equation,

$$
\sqrt{\left(a / r_{E}\right)^{2}-1}-\sec ^{-1}\left(a / r_{E}\right)=\frac{2 \pi\left(n+\frac{3}{4}\right)}{\sqrt{l(l+1)}},
$$

where $r_{E}=\sqrt{l(l+1) /(2 m E)}$ is the conjugate point and $a$ is the radius of the dot. Instead of solving the Schrödinger equation directly, the energy levels can be determined easily by the algebraic equation in Eq. 27. In the semiclassical limit, the conjugate point is close to the origin, i.e., $a / r_{E} \gg 1$. The approximate expression can be further simplified,

$$
E_{n, l} \approx \frac{\pi^{2}}{2 m a^{2}}\left(n+\frac{3}{4}+\frac{l^{\prime}}{2}\right)^{2}
$$

where $l^{\prime}=\sqrt{l(l+1)}$.

Notice that this problem can be solved exactly by the spherical Bessel functions. The hard-wall boundary requires the wave function vanishes at the surface of the sphere, $j_{l}(\sqrt{2 m E} a)=0$, that leads to quantized energy levels. In the same limit $a / r_{E} \gg 1$, the spherical Bessel function is approximated by the asymptotic expansion that leads to

$$
E_{n, l}^{e x} \approx \frac{\pi^{2}}{2 m a^{2}}\left[n+\frac{l}{2}\right]^{2}
$$

The above exact result does not seem to agree with Eq. (28) at first glance. However, if the angular momentum is also semiclassical $(l \gg 1)$, the last term in Eq. (28) is $l^{\prime} / 2 \simeq l / 2+1 / 4$ up to $O(1 / l)$ corrections. It is then clear that both give the same result. We emphasize again that the agreement is only possible when the appropriate scattering phase is included.

Another way to obtain the modified EBK quantization rule for the $1 / r$ potential is the conventional Langer's correction approach. Instead of including the appropriate scattering phase, one can obtain the same energy spectrum by modified the potential appropriately. While both approaches give the same spectrum, it is known that the wave functions calculation in scattering phase approximation is more accurate. 16, 17]

\section{MIRROR PROJECTION}

In the previous section, we treat the hard-wall boundary as depletion of the Hilbert space. An alternative way is to view it as a discrete $\mathcal{Z}_{2}$ gauge symmetry of the wave function

$$
\psi(x)=-\psi(-x) .
$$

The minus sign is chosen here to make the wave function vanishes at $x=0$ so that the boundary condition $\psi(0)=0$ is always satisfied. Since the propagator can be written down as the summation of eigenfunctions,

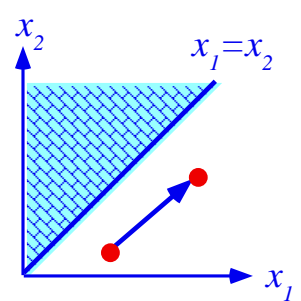

(a)

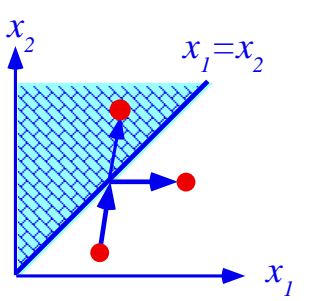

(b)
FIG. 3: Classical trajectories of two particles whose quantum statistics is replaced by the equivalent hard wall at $x_{1}=x_{2}$ in the configuration space. In part (a), a direct trajectory is shown and, in part (b), the shown reflected trajectory is equivalent to exchanging two particles which results in an extra Berry phase.

$G\left(x, x^{\prime} ; T\right)=\sum_{n} \psi_{n}(x) \psi_{n}^{*}\left(x^{\prime}\right) \exp \left[-i E_{n} T\right]$, where $\psi_{n}(x)$ is the eigenfunction with eigenenergy $E_{n}$. The discrete gauge symmetry of the wave function implies that the quantum propagator has the symmetry

$$
G\left(x, x^{\prime} ; T\right)=-G\left(x,-x^{\prime} ; T\right) .
$$

Now choose both $x=r$ and $x^{\prime}=r^{\prime}$ to be positive, the propagator can also be viewed as the wave function $G\left(r, r^{\prime} ; T\right)=\psi_{r^{\prime}}(r, t)$ that satisfies the Schrödinger equation with a delta function source at $(x, t)=\left(r^{\prime}, 0\right)$. The propagator $G_{0}\left(r, r^{\prime} ; T\right)$ without the hard-wall boundary satisfies exactly the same differential equation except that the boundary condition at $x=0$ is not met. Notice that the mirrored propagator $\bar{G}_{0}\left(r, r^{\prime} ; T\right)=G_{0}\left(r,-r^{\prime} ; T\right)$ satisfies the Schrödinger equation without the source term since the delta function $\delta\left(r+r^{\prime}\right)=0$ for positive coordinates. Therefore, the propagator that satisfies the correct boundary condition is constructed as

$$
G\left(x, x^{\prime} ; T\right)=G_{0}\left(x, x^{\prime} ; T\right)-\bar{G}_{0}\left(x, x^{\prime} ; T\right) .
$$

The above result is equivalent to Eq. (20). It is obvious that the discrete gauge symmetry in Eq. (31) is satisfied. This method is just the familiar mirror charge trick in the classical electromagnetism.

Since we can solve the hard-wall boundary by discrete gauge symmetry, we might as well go the other way around. It is possible to replace the quantum statistics between particles by the hard-wall boundaries. Let us consider the simplest case - two interacting particles with either bosonic or fermionic statistics. The discrete gauge redundancy is

$$
\psi(x)=e^{i \phi} \psi(-x),
$$

where $x \equiv x_{1}-x_{2}$ is the relative displacement between two particles. The phase correction is $\phi=0$ for bosons and $\pi$ for fermions. The discrete gauge symmetry is removed by imposing a hard wall $x_{1}=x_{2}$ in the configuration space, and a Berry phase $\phi$ accumulates upon each reflection due to the hard wall. 
Classical trajectories are classified into two categories - the direct path and the reflected one as shown in Figure 3 . If we extend the reflected trajectory into the unphysical regime inside the hard wall, as shown in Fig. 3(b), the reflected trajectory is equivalent to an exchange between two particles. This approach would be useful when studying few interacting quantum particles, e.g., two strongly interacting bosons or fermions bouncing back and forth between two hard walls. In the semiclassical limit, we can safely ignore the quantum statistics by solving all classical trajectories inside a specific triangle in the twodimensional configuration space.

\section{CONCLUSIONS}

In this paper, we study the scattering phase of classical trajectories due to sharp confining potentials. Inclusion of the energy-dependent scattering phase, the modified EBK quantization rule is derived. We also relate the hard wall boundary approach to the quantum statistics and the discrete gauge symmetry. Unlike the WKB approximation that is only applicable to smooth potential profiles, the new quantization rule provides us with an easy way to estimate the energy levels in the presence of both smooth and sharp confinement potentials.

We thank Darwin Chang for fruitful discussions, especially on the mirror projection and the discrete gauge symmetry. This work was supported by the National Science Council of Taiwan, R.O.C..

\section{APPENDIX A: POISSON SUMMATION FORMULA}

Poisson summation formula provides a convenient way to related two infinite summations together. Let us con- sider a physical system on a finite ring with length $L$ and lattice constant $a$. The total number of sites is $N=L / a$. The discrete version of the usual delta function is

$$
\sum_{x=n a} e^{i k x}=\left(\frac{L}{a}\right) \sum_{G=2 n \pi / a} \delta_{k, G}
$$

where $G$ is the reciprocal lattice vector. Consider the following summation,

$$
\sum_{n} f(n a)=\int \frac{d k}{2 \pi} F(k) \sum_{x=n a} e^{i k x}
$$

where $x_{n}=n a$ and $F(k)$ is the Fourier transformation of $f(x)$. With the help of the identity in Eq. A1 , the summation over coordinates is turned into another summation over reciprocal momenta. Taking the thermodynamical limit $L \rightarrow \infty$, the discrete delta functions are related to the continuous ones by $L \delta_{k, G}=2 \pi \delta(k-G)$. Finally, we arrive at the useful Poisson summation formula,

$$
\sum_{n} f(n a)=\frac{1}{a} \sum_{n} F\left(\frac{2 n \pi}{a}\right) .
$$

[1] R. P. Feynman and A. R. Hibbs, Quantum Mechanics and Path Integrals (McGraw-Hill, New York, 1965).

[2] Hagen Kleinert, Path Integrals in Quantum Mechanics, Statistics, and Polymer Physics (World Scientific, Singapore, 1995).

[3] M.A. Sepulveda, S. Tomsovic, and E.J. Heller, Phys. Rev. Lett. 69, 402 (1992).

[4] R.S. Manning and G.S. Ezra, Phys. Rev. A 53, 661 (1996).

[5] G. Stock and M. Thoss, Phys. Rev. Lett. 78, 578 (1997).

[6] J.H. Van Vleck, Proc. Natl. Acad. Sci. 14, 178 (1928).

[7] M.V. Berry and K.E. Mount, Rep. Prog. Phys. 35, 315 (1972).

[8] M. C. Gutzwiller, Chaos in Classical and Quantum Mechanics (Springer-Verlag, New York, 1990).

[9] M.C. Gutzwiller, J. Math. Phys. 12, 343 (1971).
[10] V.P. Maslov and M.V. Fedoriuk, Semiclassical Approximations in Quantum Mechanics (Reidel, Dordrecht, 1981), English translation.

[11] J.J. Sakurai, Modern Quantum Mechanics (AddisonWesley, New York, 1994).

[12] A. Einstein, Verh. Dtsch. Phys. Ges. 19, 82 (1917).

[13] M.L. Brillouin, J. Phys. (Paris) 7, 353 (1926).

[14] J.B. Keller, Ann. Phys. (New York) 4, 180 (1958).

[15] M.J. Lighthill, Introduction to Fourier Analysis and Generalized Functions (Cambridge Univ. Press, New York, 1960).

[16] H. Friedrich and J. Trost, Phys. Rev. Lett. 76, 4869 (1996).

[17] H. Friedrich and J. Trost, Phys. Rev. A 59, 1683 (1999).

[18] H.-H. Lin, research in progress (unpublished). 